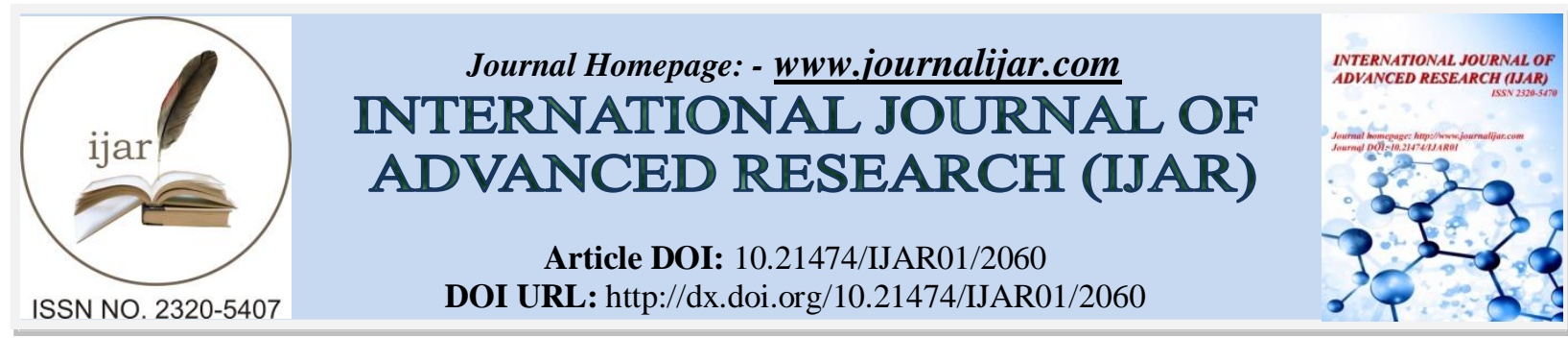

RESEARCH ARTICLE

\title{
PHYSICAL ENERGETICS OF PHOTONS (EFhEF ENERGETICS) DERIVED OF HYDROGEN ENERGETICS IN SOLAR PLASMA IS THE ENERGETICS OF SPACE CIVILIZATION OF MANKIND.
}

Ostapenko V. A.

Acad. of Ukraine Academy of Sciencses, Kiev.

\section{Manuscript Info}

Manuscript History

Received: 23 September 2016

Final Accepted: 25 October 2016

Published: November 2016

Key words:-

physical energy, EFhEF, organics, photosynthesis, decomposition, life support, current sheet, solar flare, optical spectra, the Sun, turbulence, zone boundaries, multichannel photometry, hydrogen negative ions.

\section{Abstract}

The new type of energy that is Eco Physical hydrogen Energy of Photons (EFhEF energy) is introduced. This allows the clear classification of kinds of energy: chemical (molecules), physical (atoms) and quantum (nuclear). Physical energy of photons is hydrogen energy that is the most common element in the Universe. The author performed the most comprehensive calculation of hydrogen plasma in the annex to the active (flare) processes in the solar atmosphere, which revealed the managed nature of the process of energy realization of hydrogen negative ions in plasma volume and the ability to generate this energy by man in the laboratory. Continuous radiation of negative hydrogen ions is a new (ecologically clean) type of energy that has not so far been used on Earth in such capacity. The terrestrial wildlife (for billions of years already) has benefited of this physical energetics (of hydrogen negative ion) in the form of individual photons of radiation of the photosphere of the Sun. Successful development and inheritance of the experience of managed organic synthesis (of air $\mathrm{CO}_{2}$ ) allows us to engage in the full cycle of autonomous survival.

The main problem of physics of solar flares has been solved. Magnetic field energy is a primary source of active processes in solar plasma. Current sheets (CS) of S. I. Syrovatskij are the mechanism of magnetic energy transformation into plasma energy. The first current sheet in the Nature was discovered in flare spectrum, calculated and researched experimentally. The current sheet is not discovered in a flare, as expected. As a matter of fact, solar flare is itself a current sheet, its direct observed development in chromosphere plasma. Continuous current sheet radiation is the «black» (csBLF) and the «white» (csWLF) flare (like light of Sun photosphere). It is the negative hydrogen ion excitation upon reaching hydrogen plasma density of $>510^{17} \mathrm{~cm}^{-3}$.

The results are obtained on the base of: 1 . Observations of the spectra of solar flares with the solar telescope with echelle-spectrograph and their photometric analysis with the 40-channel CCD densitometer MF4A. 2. The macro-turbulent plasma theory of spectral lines profiles of 3D- entities with intense directional plasma flows. 3. The decision of the full (with no limit on the number of quantum levels) system of stationary equations for discrete radiation and transfer equations for 
continuous radiation of the hydrogen negative ion. The task is solved to 50 flares throughout the range of their capacities. 4. At the same time the problem of the Layman-alpha radiation in the solar atmosphere is solved. The field of Lyman-alpha radiation behavior plus current sheets physics allowed finding physics of various zones of the solar atmosphere.

The application of new hydrogen energetics in order to reduce the impact of global disasters, solve (domestic and global) environmental problems, decompose the organic matter of wild-life to produce primary energy sources, create overall life sustaining systems for self-contained settlements is discussed. First installations for organic matters (and other hydrogen-containing compounds) decomposition into their components are designed. This in essence will solve the main problem of alternative energetic which is the extraction of primary energy resources from renewable deposits of wild-life.

Copy Right, IJAR, 2016,. All rights reserved.

\section{Introduction:-}

"The man will not remain forever on the Earth, but in the pursuit of light and space, it at first timidly will go beyond the Earth's atmosphere, and then win all around the solar space" (https://ru.wikipedia.org/wiki/) - predicted school teacher, scientist and inventor (Kaluga, Russia) K. E. Tsiolkovsky (5 (17).09.1857-19.09.1935). Mankind has already entered the cosmic era and now it is important to have the appropriate energetics and technologies environmental and unconditionally the full cycle of life activity of entire settlements. Where to take this energetics? Logically, it needs to find in the cosmos. " ... it is reasonable to study hydrogen processes that occur on the Sun, learn how to apply them at Earth and adjust already designed energy and transport fossil-fuel powered technologies to work on hydrogen - the most ecologically clean type of fuel» (P. L. Kapitsa, 1975 in the J. of Experimental \& Tech. Phys., Moscow). Namely this way, the author had gone for 50 years of investigating the active processes in hydrogen solar plasma with magnetic fields. In the result, the Eco Physical hydrogen Energetics of Photons (EFhEF energetics) is arisen.

Already the first author article as the student-astrophysics were devoted of the MF-4 photometry of the radiation of hydrogen $\mathrm{H}_{\varepsilon}$ and $\mathrm{H} \& \mathrm{~K}$ CaII spectral lines in solar entities with the task of dividing thermal and turbulent motions in solar events (Kurochka \& Ostapenko, 1967). During 50 years are resolved the principal conclusions are based: a) on the combined observational data of the flares on the HST-1980 (horizontal solar telescope with the echelle spectrograph); b) on spectra photometry data with the 40-channel MF4A microphotometer that created specially; c) on the theory of spectral lines profiles of macro-turbulent (convective) chaotic and directed along force lines (along arch systems) that developed specifically; d) on combined theoretical analysis of physical processes in active formations from the solving the complete system of equations statistical equilibrium for discrete emission lines of the hydrogen atom and the continuous emission of negative ion of hydrogen. The problem has been resolved without limitation of number of quantum levels. The problem of the Lyman-alpha radiation is resolved.

Beginning with the IGY-1957 of active study of solar flares close relationships between flares and magnetic fields have been established well. Here, we must to remind the efforts of Severny A. B. (CrAO, Crimea, in $1960^{\text {th }}$ years) and Syrovatskij S. I. (FIAN, Moscow, in $1970^{\text {th }}$ years). In $50^{\text {th }} \& 60^{\text {th }}$, the large solar flares were seen as an increases in brightness of the chromosphere only, which occupy large areas (up to $10^{-3}$ of the visible hemisphere of the Sun) and often in the form of two ribbon flares. Only indirect spectral and temporal data on flares pointed to the close linkage of this phenomenon with magnetic fields. These ribbons are located in areas of magnetic fields of opposite polarity. Profiles of spectral lines in the models of spatial heterogeneous entities, as well as detailed theoretical calculation of radiation of solar flares of varying power, convincingly reiterated the magnetic nature of flare events. All these international studies of the previous 50 years showed that the most likely mechanism of flare occurrence may to believe the current sheets. Namely the CS is able of to provide the conversion of magnetic fields energy in the energies of plasma radiation, accelerated plasma flows, and charged particles. The real existence of the current sheets in the Nature proven in result of directed researches by Ostapenko $(2011,2012)$. 


\section{The Origin of the EFhEF energetic:-}

The physical energetics of photons has been discovered by the author as the result of the theoretical and experimental research of active processes in the solar plasma [4, 5]. The basis is the creation of ways of getting of "solar" photons in any laboratory. The arisen of new EFhEF energetics has made a practical basis for classification of the energetics on types: the energetics of molecules (chemical energetics), the energetics of atoms (physical energetics) and the energetics of nuclei (quantum energetics). Physical energetics can be used in conjunction with chemical and quantum one on the Earth, but rather, only with the quantum energetics beyond the Earth.

The terrestrial wildlife (for billions of years already) has benefited of this physical energetics (of hydrogen negative ion) in the form of individual photons of radiation of the photosphere of the Sun. Wildlife uses all of the physical properties of atoms (known and unknown for us) for the creation and maintenance of the vital functions of organisms (flora and fauna). Successful development and inheritance of the experience of managed organic synthesis allows a person to engage in the full cycle of autonomous survival. We should follow the experience of the Nature. In the first place, this is photosynthesis.

Physical energetics can solve the problems of ecology and the replacement of fossil energy sources, which means a fundamental solution to the basic environmental problems of the energetics that is the problem of replacing of fossil energy sources. To succeed, we need to solve two major problems. It is must create reliable ways to guaranteed possession of photon field in the reactor of any installation. Then, namely the physical energetics of photons will be the basis of all inheriting technologies of wildlife. Complete self-sufficiency isolated settlements means guaranteed return for subsequent use all $100 \%$ organic waste in the form of consumption foods. Synthesis of new organics in the Nature is performed with the set of specialized molecular machines (MM), which are unrealistic to create in the first stage. But a number of problems with destruction/synthesis of the organics achievable today, using the experience in the application of catalysts to various treatments. This, the second task requires 3D-modeling and nanotechnology manipulating with thin (about the size of atoms) layers of metals during the creation of catalysts.

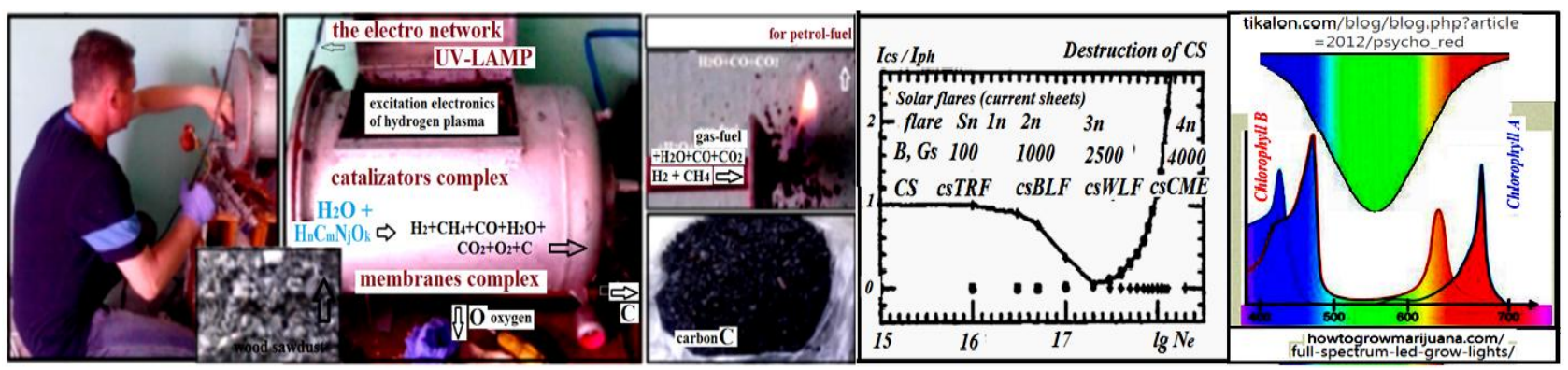

Fig.1:- Managed physical energy of hydrogen plasma in laboratory.

A. The laboratory installation of the decomposition of organic matter and the production of hydrogenhydrocarbons gas-fuel. The installation is equipped with next systems: (a) electronics for generation of "solar" photons; (b) generation of protons, thermal and accelerated electrons; (c) systems of water photolysis; (d) catalysts systems for decomposition of organics and synthesis of new organic compounds; (e) membrane systems for selection of some gas components. On the figure (left) Igor V. Ostapenko is Director of the laboratory of EFhEF- energy.

B. The calculated intensity curve of energy release in the photons form of hydrogen plasma. It is defined only by the plasma density. The curve has reflected of all solar flares emission. So, the main problem of solar flares is proved to be resolved by Ostapenko [4, 5].

C. The corpuscular-wave duality in the absorption curves of the chlorophyll molecule:

(a). UV photons excited of carbon atoms electrons accumulating energy of these photons in the C-H links of organic molecules.

(b). IR photons (waves already) heated only organic molecules (increasing the molecule's controllability).

(C). Our devices for decomposition organics into separate atoms were tested on the example of agricultural chemicals and mixtures of organic substances. They gave good results of decomposition purity (99.9\%) without visible signs of presence in the rest of the raw materials or the emergence of new compounds. The decomposition results have been verified by the government laboratories. Analysis of the composition of the synthesis-gas samples $S_{1} \& S_{2}$ are 
made in 2011 by the Gas Analytical laboratory in Gas Institute of NAS of Ukraine (Kiev) that was carried out on the gas chromatographer H Agilent 6890. The results of the analysis are summarized in the table.

Substitution of fossil gas on gas from any organic waste turns out to be possible on the basis of the new energetics only and without atmospheric ejections. The first demonstrations of our laboratory installations of operating with organics $\left(\mathrm{T}<300^{\circ} \mathrm{C}\right)$ were in $2005-2007$. Compositions of destruction products are determined by government laboratories of agro-industrial complex (AIC) and the National Academy of Sciences of Ukraine. Synthesis gas (table) is a product to obtain of gas or liquid fuel. We get gas, consisting of hydrogen and carbohydrates. As fuel this mixture has a more high energy quality than even the classic fossil gas (propane-butane).

Mankind has not yet been used the physical energy of photons. The photosynthesis of plants is the primary process of organics creating (that is $\mathrm{H}_{\mathrm{n}} \mathrm{C}_{\mathrm{m}} \mathrm{N}_{\mathrm{j}} \mathrm{O}_{\mathrm{k}}$ molecules). The $\mathrm{EFhEF}$ energetics inherits the experience of photosynthesis. Temperatures in our reactors are $\left\langle 300{ }^{\circ} \mathrm{C}\right.$ ) yet and nitrous oxides and dioxins do not appear. This requires temperatures $>600^{\circ} \mathrm{C}$. And if the network of our mobile installations has installed in places of primary accumulation of waste, it is nay to decrease temps of the growth of landfills and obtaining simultaneously the gas-fuel.

\begin{tabular}{|c|c|c|c|c|c|}
\hline components & $\begin{array}{l}S_{1}, \% \text { in } \\
\text { volume }\end{array}$ & $\begin{array}{l}\mathrm{S}_{2}, \% \text { in } \\
\text { volume }\end{array}$ & components & $\begin{array}{l}S_{1}, \% \text { in } \\
\text { volume }\end{array}$ & $\begin{array}{l}S_{2}, \% \text { in } \\
\text { volume }\end{array}$ \\
\hline $\mathrm{H}_{2}$ & 16.84 & 19.94 & $\mathrm{C}_{2} \mathrm{H}_{2}$ & 0.05 & 0 \\
\hline $\mathrm{N}_{2}$ & 9.55 & 10.94 & $\mathrm{C}_{3} \mathrm{H}_{6}$ & 0.55 & 0.81 \\
\hline $\mathrm{CH}_{4}$ & 6.67 & 10.26 & $C_{3} J_{8}$ & 0.40 & 0.62 \\
\hline $\mathrm{CO}$ & 18.59 & 14.34 & $i C_{4} H_{10}$ & 0.18 & 0.27 \\
\hline $\mathrm{CO}_{2}$ & 43.20 & 36.94 & $n C_{4} H_{10}$ & 0.16 & 0.28 \\
\hline $\mathrm{C}_{2} \mathrm{H}_{6}$ & 1.45 & 2.90 & $\mathrm{C}_{2} \mathrm{H}_{5} \mathrm{OH}$ & 0.21 & 0.23 \\
\hline $\mathrm{H}_{2} \mathrm{O}$ & 1.20 & 1.01 & $\mathrm{Neo}-\mathrm{C}_{5}$ & marks & marks \\
\hline $\mathrm{C}_{2} \mathrm{H}_{4}$ & 0.85 & 1.46 & $i C_{5}$ & marks & marks \\
\hline \multicolumn{2}{|c|}{ State Gas institute - 2011} & Де & В.П. sum & 100 & 100 \\
\hline
\end{tabular}

It is impossible to inherit of photosynthesis directly at this stage. All operations in living organisms perform molecular machines (MM), which are very intricate designs from hundreds of thousands of atoms. They are running by software of DNA molecules. However, the production of fuel from waste of organic material does not require design of special MM and therefore, it is of possible. There are all prerequisites for resolving the underlying problem, the problem of energy source. It opens and a number of other useful applications (sources of «solar» light for premises and greenhouses in all circumstances on the Earth or outside the Earth) and opens the green light for the EFhEF energetics to ensure the full cycle of life.

\section{Expected possibilities of the eco physical energetics of photons:-}

The managed hydrogen EFhEF energetics enables to solve the tasks of life outside the Earth (light, heat, as well as air and water regeneration), and return in a new cycle of using $100 \%$ organic waste (with possibility of production of consumption foods from these waste). The life Nature is managed by software numeric, recorded in DNA molecules, and works with conventional terrestrial atmospheric conditions $\left(4-40{ }^{\circ} \mathrm{C}\right)$ due to the energy of these photons. The EFhEF energetics allows us to "carry out" with themselves and our native Sun at any distance in space.

The physical energetics is able to meet the challenges of life outside the Earth: lighting and heat, air and water regeneration, and return of all $100 \%$ organic waste at the new cycle of use, and even with the production of food from this waste. The ways of the food synthesis of waste we founded. This energetics is capable to inherit the technologies of photosynthesis, which use energy in the form of separate photons. The photosynthesis only is the way to capture $\mathrm{CO}_{2}$ and of Sun's energy for creating of organic structures. Note that only chlorophylls molecule capable "to see" and to capture of the air $\mathrm{CO}_{2}$ molecule and we do not understand this. The living Nature is managed by the software, recorded in the molecule of DNA, and works in conventional terrestrial atmospheric conditions (4$40^{\circ} \mathrm{C}$ ) using the energy of solar photons. The EFhEF- energetics allows "to carry out" even our Sun at any distance in the space.

First, the EFhEF energetics provides any installation of its own source of energy ("sunny" photons). This allows us to actually inherit the rich experience of the living nature to work with organic or $\mathrm{CO}_{2}$ out of the air as it makes a molecule of chlorophyll. But, in the living nature, all operation are performed with molecular machines (MM) that are the multi-complex specialized (as rule, on one operation only) systems consisted of thousand and thousand atoms. The some MM tasks we may to resolve with catalysts on the base of metals. As food, it is sufficient to give for living organism the amino acids, given to MM possibilities of organism itself to create all proteins. 

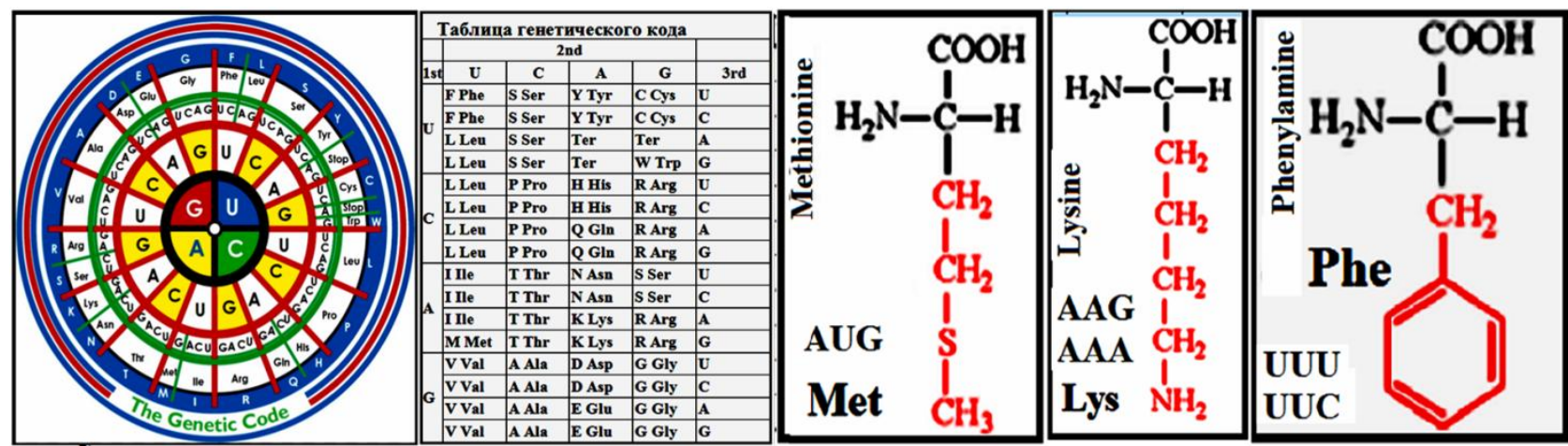

Fig 2:- The coding manner of genetic information in the DNA created on the base of nucleotides.

The table of nucleotides treads that consisted the full set of amino acids for creation of all proteins. http://www.airyourself.com/2015/05/essential-amino-acids-in-products.html

The amino-acids examples (right, 3 of 22) consist of two parts: a) standard-survival (black color) and b) functional radical (red color).

The proteins, carbohydrates, and lipids are the food of living entities. Namely the food we must to produce from waste in settlements outside the Earth. The basis structural unit of the proteins is molecules of amino acids. These are a chain of atoms, which on the one hand goes outward the hydrogen atom of the water molecule, on the other hand its hydroxyl group. In the middle are a variety of other components. Of these amino acids are going to squirrelhydrogen ion $\left(\mathrm{H}^{+}\right)$one amino acid is combined with the hydroxyl ion $\left(\mathrm{OH}^{-}\right)$other amino acids with the formation of water molecules. Among proteins there are proteins-enzymes that regulate chemical reactions in cells. And all proteins are the most important structures of living organisms.

Proteins (amino acids), carbohydrates and lipids have universal base and they are the food of living beings. It is food we have to produce, primarily from waste in remote and isolated settlements, especially outside of the Earth. Any protein is a long chain of hundreds or thousands of amino acids compiled a total of 22 different varieties. Properties and purpose of each protein is determined by the order of theae amino acids in the chain and their number. Among proteins there are proteins-enzymes that regulate chemical reactions in cells (a type of insulin) or molecular machines (tRNA type) that transport the DNA information. And all proteins are the most important structures of living organisms. All chemical and physical properties and features of protein as MM, there are already in the set of existing 22 amino acids, radical configurations (in red on Figure 2).

To link of amino acids in the chain provides the standard set of groups of atoms, assembled on the basis of the carbon atom (black on fig. 2). On the one hand the outside group $\mathrm{NH}_{2}$ and on the other hand the hydroxyl group $\mathrm{COOH}$ is located. When ion $\left(\mathrm{H}^{+}\right)$of one amino acid is combined with a hydroxyl ion $\left(\mathrm{OH}^{-}\right)$of other amino acids, the amino acids are linked of each other with formation of water molecules. All instructions on protein assembly recorded in the program of the DNA molecule, which begin to work when merging two special "cells" (complementary sub-spirals (man' and women') in one dual-spiral of the DNA program. The biomass of new body increased of cells number by their dividing in half. To create the full structure of the organism (human body is $10^{14}$ cells), each cell must "see" their maneuver and designation at any stage of creation and the evolution of life. It is probably possible only when the DNA program included and stereo picture-matrix of whole body. Then it becomes clear and managed failover of the mode larva-butterfly that clearly runs under the two sub-programmes of the DNA.

So, all food consists of amino acids, carbohydrates (glucose) and fat (membrane and energy reserves). All initial set of these raw materials is produced by plants of $\mathrm{CO}_{2}$, water with energy of photons. This is and our way of feeding isolated settlements outside of the Earth. Ways to obtain photons are found; photosynthesis and experience of living nature can and should inherit.

\section{The Full calculation of hydrogen plasma for solar entities:-}

The common calculations of discrete emission (in spectral lines) of hydrogen atoms and the continuous emission of hydrogen negative ions are made. The equation system (UST) of statistical equilibrium has been resolved in most full manner for radiation of hydrogen plasma in solar active formations. As initial data we use the observed intensities of spectral lines of hydrogen plasma. We use also all complex of modern information on solar flares and 
other flare-like formations and events. In the first time, the full system of equation of statistical equilibrium for hydrogen plasma without limiting of number of quantum states has been composited. We found also whole set of probabilities of elementary processes. We have outlined the physics of solar flares and flare-like events In the complex with analysis of spectral lines profiles and diffusion of radiation in turbulent (in fact, convective plasma). We have discovered that the energetics of hydrogen negative ions is by managed and it may be recreating in the laboratory. There is physical energetics of photons. Namely this energy from Suns' photosphere is used for ecological creation and operation with the organics on the Earth by life' Nature. We obtained also the real possibilities of creation of hand-made sun for people in any conditions on the Earth and outside the Earth.
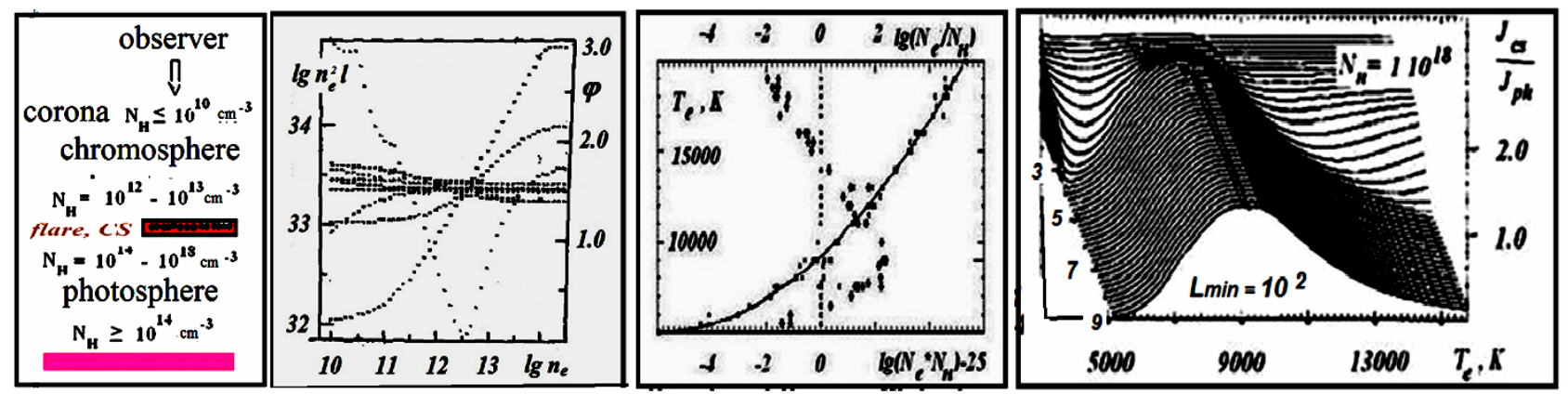

Fig 3:- Some calculation results of hydrogen plasma for discrete and continuous radiation $[4,5]$.

a. The model of a flare and a compression CS layer in solar chromosphere.

b. The decision of the UST system of statistical equilibrium as $\mathbf{n}_{\mathbf{e}}=\mathbf{f}\left(\mathbf{n}_{\mathbf{e}}{ }^{2} \mathbf{l}\right)$ dependence.

c. The $\mathrm{N}_{\mathrm{e}} / \mathrm{N}_{\mathrm{H}}(\mathrm{e})+\mathrm{N}_{\mathrm{H} /} \mathrm{N}_{\mathrm{e}}(\mathrm{H})$ ratios and $\mathrm{N}_{\mathrm{e}} * \mathrm{~N}_{\mathrm{H}}$ (asterisk) depending on the plasma temperature.

d. The continuous radiation of hydrogen negative ions in the model of compressed layer for the csWLF stage of the cs(TRF-BLF-WLF) evolution when CS plasma density $\mathrm{N}_{\mathrm{n}}=510^{18} \mathrm{~cm}^{-3}$ and the set of values of temperature $\mathrm{T}_{\mathrm{e}}=(5-15) 10^{3} \mathrm{~K}$ and its stratum along the beam of the $\mathrm{L}_{\mathrm{cs}}=1+0.2 \mathbf{m}($ where $\mathbf{m}=1-40)=\left(10^{9} \mathrm{~cm}-10 \mathrm{~cm}\right)$.

The Lyman series is the series of energies required to excite an electron in hydrogen from its lowest energy state to a higher energy state. The case of particular interest for cosmology is where a hydrogen atom with its electron in the lowest energy configuration gets hit by a photon and is boosted to the next lowest energy level. The energy levels are given by $E_{n}=-13.6 \mathrm{eV} / \mathrm{n}^{2}$ and the energy difference between the lowest $(\mathrm{n}=1)$ and second lowest $(\mathrm{n}=2)$ levels corresponds to a photon with wavelength $1216 \AA$. The reverse process can and does occur as well, where an electron goes from the higher $n=2$ energy state to the ground state, releasing a photon of the same energy.

The temperature of Lyman-alpha radiation $\mathrm{T}_{21}$ is important to know when physical conditions in the emission formations are found by the solution method of UST equations system of the statistical equilibrium. This is the problem of assessing the one of main parameters of the model, which is the plasma density, which do not found by observations from the Earth' surface.. When the temperature is $<8000 \mathrm{~K}$ the majority of hydrogen atoms is located in the ground quantum state. All transitions on the first level are accompanied by radiation in the UV region. At the same time, the relationship between the electron temperature $T_{e}$ and temperature $T_{21}$ is unknown. Any researcher must to consider of the $T_{21}$ as a free parameter (Sobolev, 1961). We may to use the two extremely cases. The A case when optical thicknesses is zero in the Lyman series; Secondly, this is the B case: the LTE conditions are in the Lyman lines (Baker \& Menzel, 1938). We believe physical parameters by homogeneous and without change along the line of sight. In calculations used the hydrogen atom model with the levels number $m=9$ and continuum.

Later, in the full UST equations system is conveniently used the (24) approximate expression, which has replaced

the equation for the first level: $\mathrm{T}_{21}=7300+\frac{T_{e}-7300}{1+e^{-x}}$,

$$
\mathrm{x}=\lg n_{e}^{2} l-29.4-3.14\left(\lg \mathrm{T}_{\mathrm{e}}-3.7\right) .
$$

We analyzed detail (Ostapenko, 2011; 2012, table 4.5.1) the energy balance is given for transitions between the quanta levels for the flares of all power. The total energy of flares is provided by own thermal excitation mechanism (90\%) with regard to the fields of Sun' radiation (10\%). Most energy $(60 \%)$ radiates in the Balmer series. The Balmer continuum, which is usually not stand out against the photosphere background, emitted to $45 \%$, and in the intensities line $\mathrm{H}_{\square}$ in flares is $20 \%$ of the whole energy of the Balmer series. 
Self-agreed data about the physical state of the flare plasma have been computed from solutions of equations system (UST) of the statistical equilibrium for the linear/discrete radiation. Similar separate and not-agreed data about physical parameters for chromosphere, prominences, and flares are available. We for the first time have obtained the homogeneous data about the flares and immediately across the all range of their capacities. As the input data we used the observed intensities of Balmer emission lines of hydrogen atom. The problem of complete and reliable observed data is solved. The compiling and solving method of the UST system is developed without limiting the number of quantum levels. The problem of self-agreed probabilities for all the elementary processes of ionization and excitation of hydrogen atom is solved. The problem of Layman radiation field is solved to be important for the correct assessment of the number of hydrogen atoms in the ground quantum state. The terrestrial observations are not allowed to receive the data in UV-range. We used for the first time the real expression for Lyman-alpha radiation.

We have obtained the solution for 50 solar flares of varying capacity. With all faithfully to these values the physical conditions in the approach of the model, these characteristics have a distant relationship to the real physical conditions in flares. The whole question is consisted in the complete inadequacy between by the actual conditions of the formation of hydrogen spectral line profiles for solar flares and by models of horizontal layer. Solar flares in optical range are 3D-formations. They are of loop systems or entire arcades with intense plasma flows. The same behavior of «physical» and geometrical characteristics of the object is understood namely in the flare arch model. It is impossible to be confident in the correctness of conclusions not only on physical conditions, but even about mechanisms of flare plasma excitation.

\section{Physical peculiarities of various external zones of solar atmosphere:-}

The solution of the problem of the Layman-alpha radiation field in the solar atmosphere simultaneously with discovery of the current sheets (CS) in the Nature (on the Sun) have allowed by the author to revise both the available models of solar atmosphere and the heat nature of the corona. We distinguish four zones now in the external solar atmosphere, in their sequence and in their physical sense: photosphere, chromosphere, transition zone and corona. Now, it is believed that coronal plasma temperature has exceeded of million degrees. Our results indicate the structure of the corona composed of multiple non-thermal jets of the CS that change the very notion of the term of temperature. The corona is transparent in all hydrogen lines of the Lyman series (the A case of Menzel approximation) and radiative processes take only place in excitation of hydrogen plasma.

Solar plasma of external atmosphere demonstrated clear the macro-turbulence and intensive mass flows as it have been found on the research base of solar plasma formations changed dramatically the conditions of radiation transfer. The theory of spectral line profiles for flare and flare-like events, which used the model of turbulent and structuring plasmas allows author to describe correctly of all observed species of all flare-like events on the Sun. All types of plasma motions so as micro and macro turbulent as well as the directed flows are researched on the background of atom thermal movements. The theory of the spectral line profiles for 3D-structures such as the arch systems with intensity plasma flows has been created. The methods of radiation diffusion for homogeneous plasmas that have been created earlier we need to review, as the turbulent medium changes radically the length of free path of photons. The using of average self-absorption factors allows us to have efficient getting ways of physically correct results.
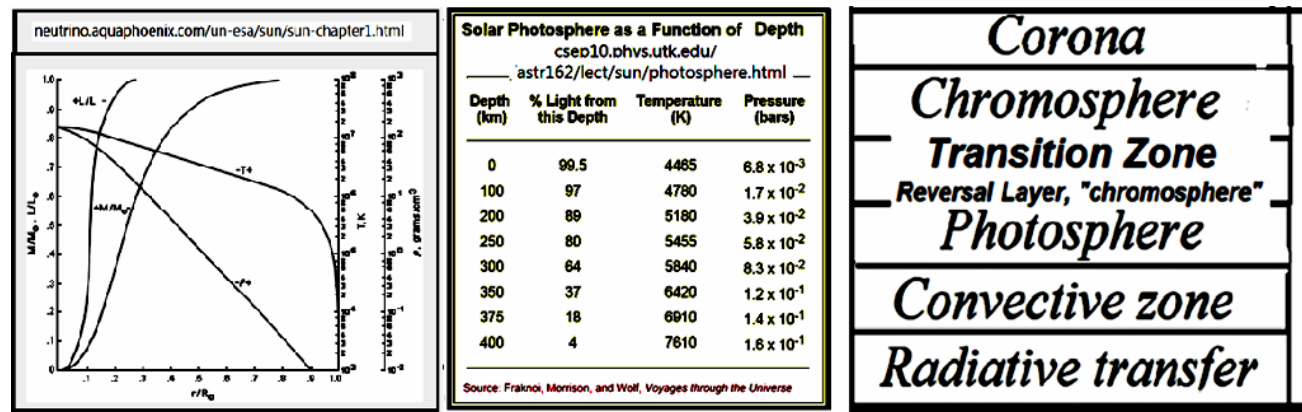

$$
\begin{aligned}
& N_{H}=10^{10} \mathrm{~cm}^{-3} \\
& N_{H}=10^{14} \mathrm{~cm}^{-3} \\
& N_{H}=10^{18} \mathrm{~cm}^{-3} \\
& N_{H}=10^{18} \mathrm{~cm}^{-3} \\
& N_{H}=10^{22} \mathrm{~cm}^{-3}
\end{aligned}
$$

Fig.4. The standard model and physics of external zones of the Sun.

a. A standard solar model of the present solar interior: $\rho \mathrm{c}=158 \mathrm{~g} \mathrm{~cm}-3, \mathrm{Tc}=1.57107 \mathrm{~K}$.

b. The depth of the photosphere (Physical encyclopedia.mht) - it is believed this is $\sim 300 \mathrm{~km}$.

c. Physics of the external zones is determined be the plasma density $[4,5]$. 
The standard model of the Sun (hence, other stars) needs are likely to be revised in the theory of convective plasma movements as analyzed by the author with regard to solar inner layers. Mean free path (probability of photons escape) dramatically increases in a turbulent plasma and photons will diffuse to the solar surface is much faster (not many thousands of years). Probability curves are the release of photons and curves of half-widths of spectral lines (Doppler widths or most probable velocities). But these methods cannot distinguish between radiative and convective zone apart. To this end, we must use the methods of detail energy transfer.

The model calculations of the linear hydrogen plasma emission are produced by the author for the horizontal compacted stable chromosphere layer. The results confirm good traditional ideas about the physical condition of chromosphere flare plasma in the optical range due similar ground model representations. Observed line profiles and mechanisms of their broadening, including the influence of radiation transfer effects in the same model, in general, are placed in the overall scheme of these representations. The linear hydrogen emission is provided by thermal excitation mechanism (electron impacts and photosphere and own emission fields).

The absence of the profile flat tops with significant optical thicknesses is due to the influence of intensity turbulence in emission volume. The theory of the radiation transfer in homogeneous plasma leads to the appearance of the fade intensity in the profile top, although these profile peculiarities are smoothed by own movements of radiation elements (Ostapenko, 2012). The VAL-C models, which atmosphere are in the hydrostatic equilibrium shown the height of $\sim 10^{3} \mathrm{~km}$ comparison of height of the real solar atmosphere reaches of $\sim 10^{5} \mathrm{~km}$.

Self-agreed data about the physical state of the flare plasma have been computed from solutions of equations system (UST) of the statistical equilibrium for the linear/discrete radiation. Similar separate and not-agreed data about physical parameters for chromosphere, prominences, and flares are available. We for the first time have obtained the homogeneous data about the flares and immediately across the all range of their capacities. As the input data we used the observed intensities of Balmer emission lines of hydrogen atom. The problem of complete and reliable observed data is solved. The compiling and solving method of the UST system is developed without limiting the number of quantum levels. The problem of self-agreed probabilities for all the elementary processes of ionization and excitation of hydrogen atom is solved. The problem of Layman radiation field is solved to be important for the correct assessment of the number of hydrogen atoms in the ground quantum state. The terrestrial observations are not allowed to receive the data in UV-range. We used for the first time the real expression for Lyman-alpha radiation.

We have obtained the solution for 50 solar flares of varying capacity. With all faithfully to these values the physical conditions in the approach of the model, these characteristics have a distant relationship to the real physical conditions in flares. The whole question is consist in the complete inadequacy between by the actual conditions of the formation of hydrogen spectral line profiles for solar flares and by models of horizontal layer. Solar flares in optical range are 3D-formations. They are of loop systems or entire arcades with intense plasma flows. The same behavior of «physical» and geometrical characteristics of the object is understood namely in the flare arch model. It is impossible to be confident in the correctness of conclusions not only on physical conditions, but even about mechanisms of flare plasma excitation.

The field problems of Lyman-alpha radiation have been considered and resolved. The empirical expression is obtained, which has its own physical sense. These Lyman-alpha data with the results of the analysis of continuous radiation have allowed to install by the physical sense of different zones of the solar atmosphere (photosphere, transition region, chromosphere, and corona).

The transition zone is the place of formation of the solar wind. The transition zone is the place, where the mechanisms of energy transfer from the internal layers of the Sun are changing. Here energy of the radiative transfer is changed in energy by plasma jets out lot of small current sheets in the space between the granules. Convective elements are observed as the photosphere granulation. It was the granular structure that creates the conditions of formation by the current sheets in the photosphere-chromosphere transition zone of convective structure with its distinctive field of mass plasma flows. The new concept of transitional zone is agreed well with the current sheets available in the Sun and their direct observed manifestations in the photos as well as with the photometry using of solar wind manifestations.

The continuous emission of hydrogen negative ion in Sun's chromosphere plasma is calculated. The initial physical characteristics of chromosphere plasma are simultaneously found in result of solution of the UST equations system 
of statistical equilibrium for line emission of flare hydrogen atom in all range of their powers. The continuous emission of the CS (radiation of hydrogen negative ion) is characterized for its development by the following main parameters: a) the plasma densities $\mathrm{N}_{\mathrm{cs}} \sim 10^{14}-10^{18} \mathrm{~cm}^{-3}$; b) temperature $\mathrm{T}_{\mathrm{cs}} \sim 9000{ }^{\circ} \mathrm{K}$; c) the length along the beam $\mathrm{L}_{\mathrm{cs}} \sim 10^{5}-10^{1} \mathrm{~cm}$. The magnitude of the $\mathrm{L}_{\mathrm{cs}}$ is decreased as $\mathrm{N}_{\mathrm{cs}}$ is increased. Maximal attainable development stage of each flare is determined by the strengths of interacting magnetic fields. When the plasma densities in the CS layer are $\mathrm{N}_{\mathrm{cs}}<510^{16} \mathrm{~cm}^{-3}$ (weak flares), the CS is transparent (csTRF) for the continuous emission of the photosphere. Continuous csBLF emission («black» flares) is appeared when plasma density are $510^{16}<\mathrm{N}_{\mathrm{cs}}<710^{11} \mathrm{~cm}^{-3}$ (flares of middle power). In powerful flashes $\left(\mathrm{N}_{\mathrm{cs}}>710^{17} \mathrm{~cm}^{-3}\right)$, the csBLF is replaced by csWLF «white» emission.

A typical «white» flare occurs by individual bright knots $\sim 5000 \mathrm{~km}$. Maximal knots brightness are $\sim 175 \%$ of the brightness of the adjacent quiet photosphere. The width of the $\mathrm{H}_{\alpha}$ line profile is growing fast within 5 minutes and at the time of the appearance of flare in the integral light reaches $17 \AA$. By increasing in size, a flare takes the appearance of an arch system. Arch structures are continuously growing in the direction from the bottom to up. The csWLF flare is appeared, when the $\mathrm{H}_{\square}$ line width $>20 \AA$. The csWLF is destructed, when the $\mathrm{H}_{\square}$ line width $>40 \AA$.

Calculations using line and continuous radiation of hydrogen plasma, simultaneously with observations and spectra photometry with 40-channel MF4A, clarify all basic manifestations of flares and flare-like events in solar plasma. This proven and correctness of calculations performed.

Geometric measures of CS are $10^{5}-10^{1} \mathrm{~cm}$. An emergence of a new magnetic flux in an active region, in suitable conditions, this is the time of a chromosphere CS origin (in typical altitudes of 5-10 10 $0^{3} \mathrm{~km}$ above the photosphere) and the time of a flaring process origin. For the occurrence of flare is not required nor having a coronal current sheet, nor the process before-flare accumulation of energy. A flare is not a destruction process of the already existing coronal CS. A flare itself is the current sheet. All flare energy is provided by a CS plasma flows.

A cs(TRF-BLF-WLF) process is very fast (tens of seconds or minutes). If a current sheet is located vertically, its cumulative effect in optical images of limb flares is caused by the same effect of a CS own and shows simultaneously upward (CMEs) and downward (shock wave or tsunami).

\section{The Discovery of the current sheets in the nature:-}

We declare about the discovery in the Nature of theoretical current sheets by S. I. Syrovatskij. The first "natural" current sheet was discovered in flare spectrum, calculated and researched experimentally. The current sheets (CS) are found in chromosphere and downward levels in optical range data. The main problem of physics of solar flares has been solved. Magnetic field energy is a primary source of active processes in solar plasma. Current sheet (CS) of S. I. Syrovatskij is the mechanism of magnetic energy transformation into plasma energy. The current sheet is not discovered in a flare, as expected. As a matter of fact, solar flare is in itself a current sheet, its direct observed development in chromosphere plasma. Continuous current sheet radiation is the «black» (BLF) and the «white» (WLF) flare (like light of Sun photosphere). It is the negative hydrogen ion excitation upon reaching hydrogen plasma density of $>510^{17} \mathrm{~cm}^{-3}$. 


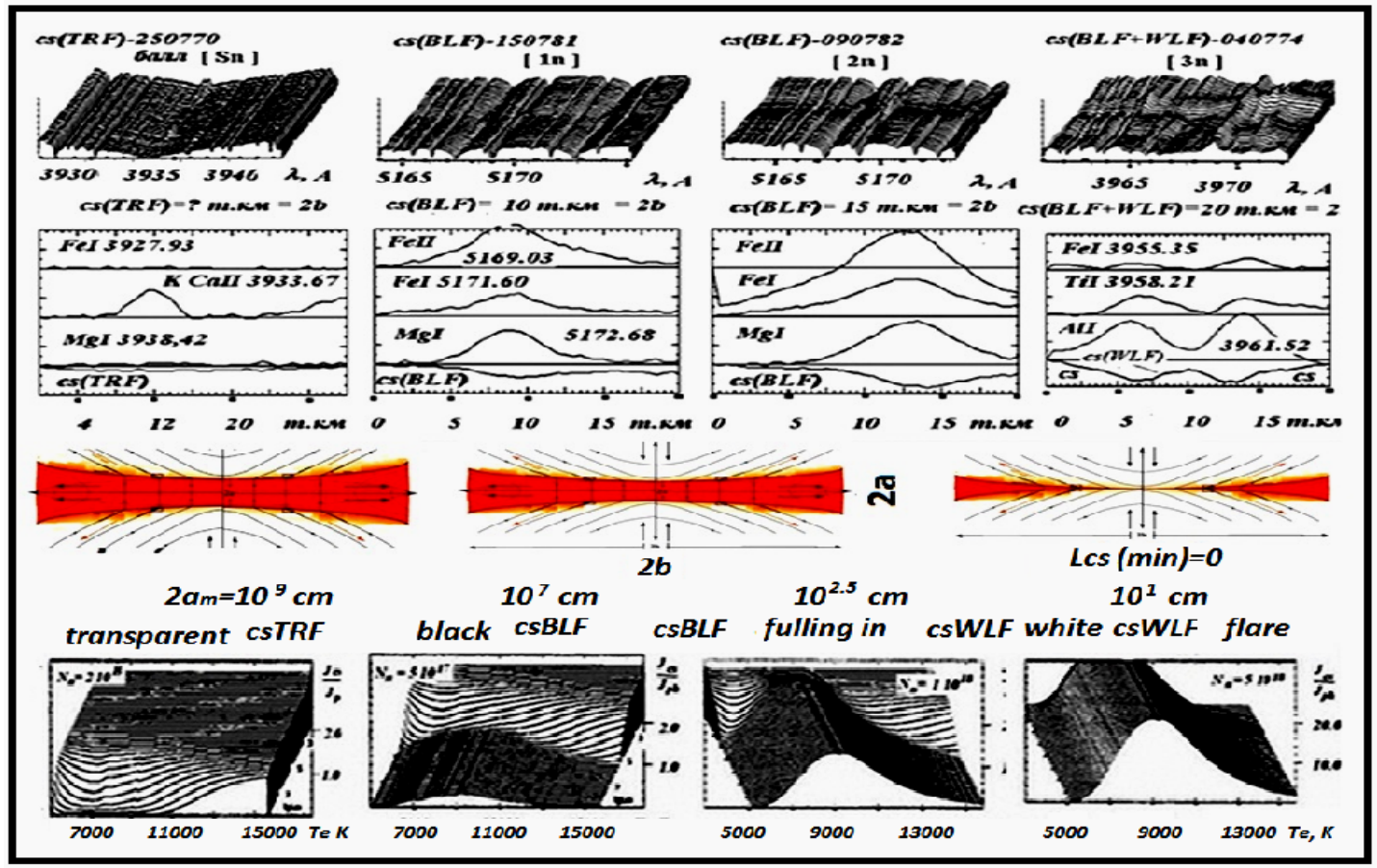

Fig 5:- The cs(TRF-BLF-WLF) development of the current sheet with the change of the flares power (Ostapenko, 1997; 2011, 2012). In top panel are the flares spectra by the MF4A photometry. Lower are the records of the same fragments along the spectral line (along the surface of the Sun). The CS layer width $2 \mathrm{~b}$ is increased while its thickness $2 \mathrm{a}$ and is decreased with increasing of flares power. The maximal plasma density it is appeared to be in the middle layer and reduced to chromosphere densities on its periphery. In the down panel we show the theoretical calculation of continuous emission of hydrogen negative ions in the CS condensed layer for set of temperatures $\left(\mathrm{T}_{\mathrm{e}}\right)$, lengths of the line of site $\left(\mathrm{L}_{\mathrm{cs}}\right)$ and plasma density $\left(\mathrm{N}_{\mathrm{cs}}\right)$.
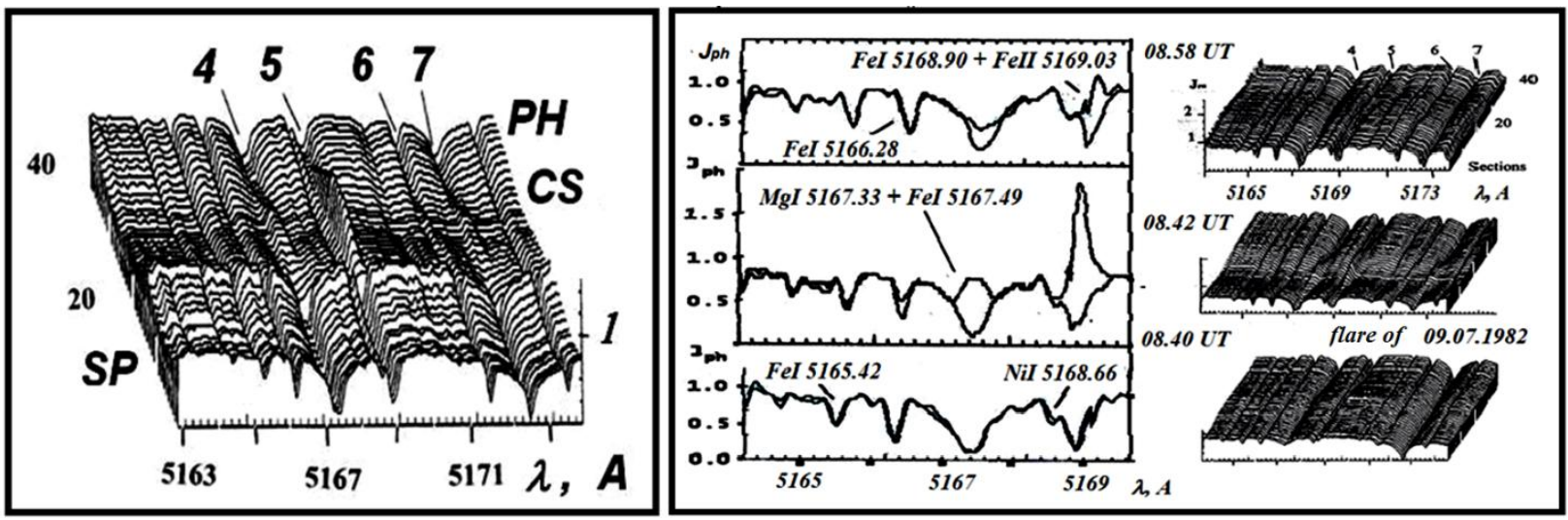

Fig 6:- Photometry with 40-chakkel MF4A of the metal lines appearance during the cs(TRF-BLF-WLF) flare development (Ostapenko, 2011).

a. Combined spectrum fragment of the disk flare on 09.07.1982 and the sunspot on 13.06.1989 in spectral lines (4)-MgI 5167.33+FeI 5167.49, (5)-FeI 5168.90+FeII 5169.03, (6)-FeI 5171.60, and (7)-MgI 5172.68.

b. The spectrum fragments 09.07.1982 solar flare (SF) on the various stages \{cs(TRF-BLF-WLF) \} of its development. In the left are given the one-dimensional scans mapped by the level of continuous spectra with spectra of the sunspot (SP) and the photosphere $(\mathrm{PH})-\mathrm{SP}+\mathrm{PH}, \mathrm{csBLF}+\mathrm{PH}$, and csWLF+PH.

The development of space researches showed that solar flares are a very wide range of processes and phenomena in all ranges of wavelengths from radio to $\gamma$-ranges on all virtually altitudes in the atmosphere of the Sun. The main part of the flare power is released in the form of kinetic energy of plasma flows of the velocities in the space up to $1000-2000 \mathrm{~km} / \mathrm{s}$. In the chromosphere lines $\left(\mathrm{H}_{\alpha}\right)$ solar flares observed as significant brightness increase of restricted areas of the solar surface. So, during many years has used the term «chromosphere flare». 
The metal lines are appeared in the compressed CS layer (fig.5, 6). Their intensity correlated with the common cs(TRF-BLF-WLF) evolution. Other words, the intensity of flare spectral lines follows to plasma density as the CS itself. When the plasma density $\mathrm{N}_{\mathrm{cs}}>10^{18} \mathrm{~cm}^{-3}$, the turbulence in the CS layer has arisen and metal lines intensity has dropped also.

Current sheets are two-dimensional magnetic plasma structures because of input plasma in a layer and output from they are in orthogonal directions (fig.7b). In strong magnetic fields the layer width (2b) is a lot more than its thickness (2a). The CS is formed in chromosphere in the place of contact of the new compact (CAS) magnetic field and pre-existing extended (EAS) arch systems in active regions as it follows yet from the first observations. Near the photosphere level (in the transition zone) the vertical CS is returned into piston due plasma density gradient (fig.7c).
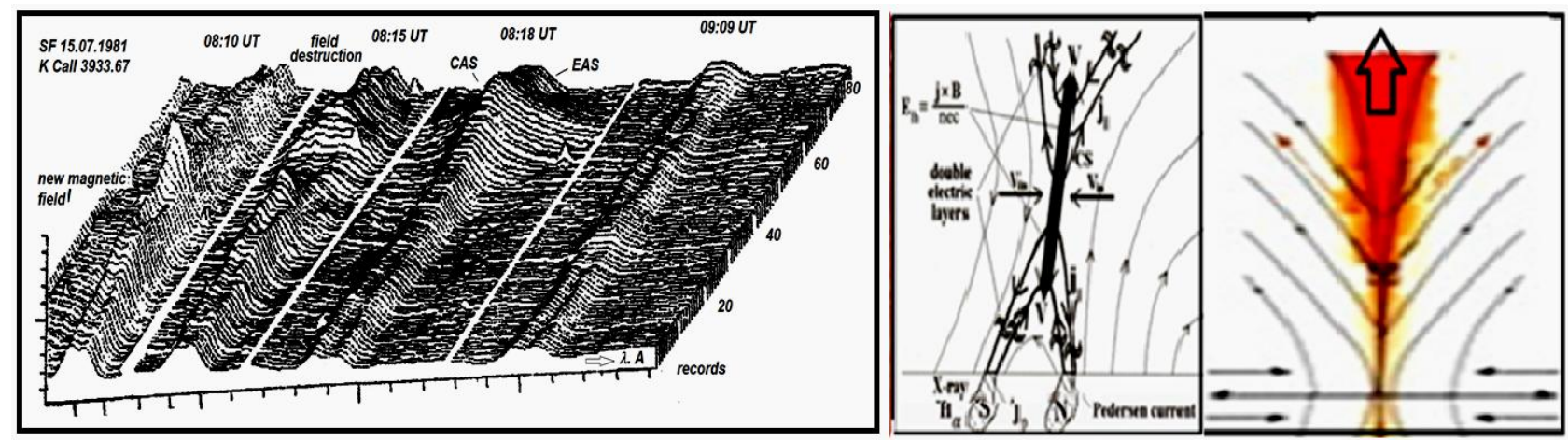

Fig 7:- The photometry with 40-channel MF4A and modeling of the CS.

a. The emergence process of the new magnetic flux (08:10 UT), destruction of the magnetic field of extend arch systems (EAS) of the active region (08:15 UT), and the formation of the compact arch systems (CAS) with the current sheet (08:18 UT) in the 15.07.1981 flare in K CaII 3933.67.

b. 1 The model of a vertical CS in the corona and the same CS in the transition zone as rule and in the chromosphere often the gradient of plasma density turns the vertical CS into a piston with one-sided ejection upward of the plasma (modeling and photometry, fig.5). (Ostapenko, 1997; 2011, 2012).

The first moment of the CS forming is detected has means already time of the flare beginning (fig.6). Own the CS compacted layer is observed in the continuous spectrum as the csBLF or csWLF flare strip (fig.7). Metals lines (many of them in radiation) are formed in the CS layer. The CAS model of the compact arch with the CS (Ostapenko, 2011) allows explaining a whole range of related flaring phenomena. So (fig.7b), the flow of plasma 4 (to upward) is registered as the surge or the radio burst of III type. The flow 2 is throwing a return ejection. The plasma flow from the CS downward is manifested as intense X-ray radiation (HXR) and specific profiles of spectral lines in the optical range (Ostapenko, 2011, 2012).

The contact place of CAS \& EAS arch systems is the so-called flare «core» in the spectrum (fig.6; 08:18 UT). Especially powerful csWLF flares occur in strong magnetic fields, when the CS plasma density $\mathrm{N}_{\mathrm{cs}}>10^{18} \mathrm{~cm}^{-3}$. Strong plasma turbulences are resulted in the CS the explosive destruction of CAS \& EAS arch tops that we see as the coronal mass ejection (CME) and tsunami as well as the huge SXR \& HXR emissions and radio IV type bursts in the corona. Thwe example of such power flare is shown on the title page of author monographs. The shock wave (tsunami) has passed over the surface of the Sun with a speed of $>600 \mathrm{~km} / \mathrm{s}$ (APOD-13.12.2006). The majority of flares never reach the stage of plasma turbulence. This fact allows us to connect the observed flare view and its power, extension wings of hydrogen and H\&K CaII lines and the presence of csWLF or csBLF emissions in CS layers with strengths of magnetic fields of the CAS \& EAS arch systems. 


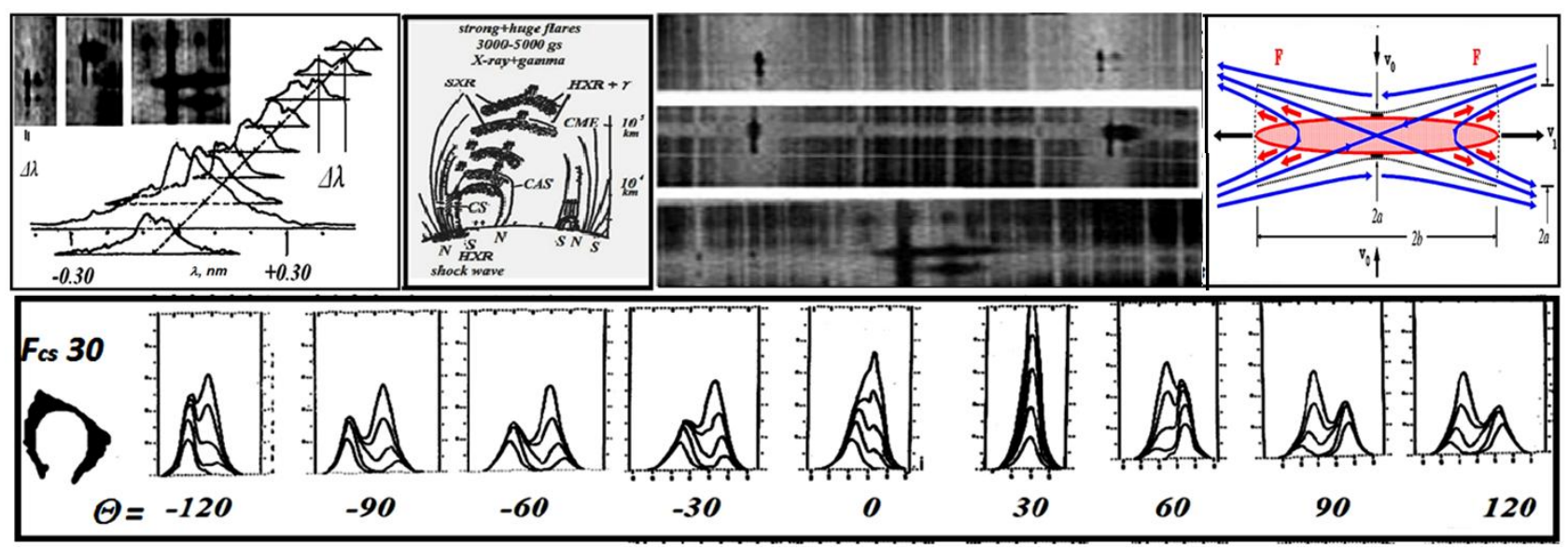

Fig 8:- The manifestations of current sheets in flare spectra and spectral line profiles are described in the one common model of the arch system (with the CS or without it).

(Lower panel): Calculated spectral line profiles of flare "cores" (plasma flow velocities are $80-250 \mathrm{~km} / \mathrm{s}$ ) or of Severny "moustache" (plasma flow velocities out of wto to up $1000 \mathrm{~km} / \mathrm{s}$ ).

(Upper panel): The flare spectrum fragments in the H or K CaII (importance Sn) 06.09.1957 faint flare (in top); the (importance 2n) 12.07.1961 big flare (in middle); and the (importance 3n) 04.07.1974 power flare (in down). The CAS is shown by both discrete emission of the broad lines of hydrogen, and narrow emission of metals lines, as well as by csBLF (or csWLF) strips radiation in the continuous spectrum. The flare «core» that is the new compact arch system (CAS) has emerged under extended arch systems (EAS) of active regions and in the contact and interaction place between CAS and EAS magnetic fields is formed the current sheet.

\section{Common Conclusions:-}

The solar flare itself is proved to be the current sheet (CS), its direct observed manifestation in the optical spectrum. Any solar flare is an observed development process of the energy magnetic field release. The CS is located in chromosphere where we see it's directly in optics. Solar flares in the chromosphere (other flare-similar formations of transitional zone) there are numerous displays of current sheets that actually are observed on the Sun. For the first time, the basic physical parameters of the real CS have been derived in the solar plasma based on observational data and complex calculations. The conditions stabilization and destruction of current sheets are found.

The current sheets (CS) are found in chromosphere and downward levels in optical range data. The main problem of physics of solar flares has been solved. Magnetic field energy is a primary source of active processes in solar plasma. Current sheet (CS) of S. I. Syrovatskij is the mechanism of magnetic energy transformation into plasma energy. The current sheet is not discovered in a flare, as expected. As a matter of fact, solar flare is in itself a current sheet, its direct observed development in chromosphere plasma. Continuous current sheet radiation is the «black» (csBLF) and the «white» (csWLF) flare (like light of Sun photosphere). It is the negative hydrogen ion excitation upon reaching hydrogen plasma density of $>510^{17} \mathrm{~cm}^{-3}$. The simultaneous calculations of lines emission of hydrogen atoms and the continuous emission of hydrogen negative ions as well as the flares spectra MF4A photometry have shown:

The magnetic nature of the primary mechanism of solar flare activity is proven. Any flare-like event is the collision process of two magnetic fields. The magnetic energy turns into the energy flows of plasma and charged particles as also as in energy of thermal radiation by means of current sheets (CS). The collision process of magnetic fields is developed until equaling magnetic and gaseous pressures $\left(\mathrm{B}=\mathrm{V}_{\mathrm{cs}} \sqrt{4 \pi m_{H} N_{c s}} ; \mathrm{B}^{2} / 8 \pi=\mathrm{N}_{\mathrm{cs}} \mathrm{kT}\right)$ in the compressed layer of the CS. The managed character of energy release of hydrogen plasma in continuous radiation is found. The main consequence of studies of solar flares is the birth of the new physical energetics, energetics of the photons (EFhEF energetics). It is this energetics should ensure the future of people: possibility of survival on the Earth in conditions of global catastrophe, the ability to provide the full cycle of life support returning in a new cycle of use of all products (including $\mathrm{CO}_{2}$ and $\mathrm{CO}$ of the air), the ability to provide the sunlight at any distance from the Sun.

The new type of energy that is Eco Physical hydrogen Energy of Photons (EFhEF energy) is introduced. This allows the clear classification of kinds of energy: chemical (molecules), physical (atoms) and quantum (nuclear). Physical 
energy of photons is hydrogen energy that is the most common element in the Universe. The author performed the most comprehensive calculation of hydrogen plasma in the annex to the active (flare) processes in the solar atmosphere, which revealed the managed nature of the process of energy realization of hydrogen negative ions in plasma volume and the ability to generate this energy by man in the laboratory. Continuous radiation of negative hydrogen ions is a new (ecologically clean) type of energy that has not so far been used on Earth in such capacity. The terrestrial wildlife (for billions of years already) has benefited of this physical energetics (of hydrogen negative ion) in the form of individual photons of radiation of the photosphere of the Sun. Successful development and inheritance of the experience of managed organic synthesis (of air CO2) allows us to engage in the full cycle of autonomous survival.

\section{References:-}

1. Kurochka L. N. \& Ostapenko V. A. On the illumination of hydrogen and CaII in chromosphere' flares. Astrophysics Questions (Kiev), 1967, p.91-95 (ru).

2. Ostapenko V. A. On the observation possibility of current sheets on the Sun. Letters to Astron. J., 1981, v.7, p.561-565 (ru). Are current sheets observable on the Sun? Soviet Astronomy Letters, v.7, 1981, p. 313-315. 2014, http://adsabs.harvard.edu/full/1981SvAL....7...3130.

3. Ostapenko V. A. Accounting of the self-absorption in spectral lines for inhomogeneous plasma. Solar Data (Pulkovo, ru), 1981, No.2, p.90-96.

4. Ostapenko V.A. Preliminary results of optical solar flare spectra studies using a 40-channel densitometer. Astrophysics \& space science. 1997, v.252, p.335-348.

5. Ostapenko V.A. Solar flares: current sheets on the Sun, ecological hydrogen energetics on the Earth. Kiev, "Ukraine", 2011, 452 p.; (ru).

6. Ostapenko V.A. Solar flares: current sheets on the Sun and ecological hydrogen energetics on the Earth. Kiev, "Ukraine", 2012, 185 p (en).

7. Ostapenko V. A. Ecological hydrogen energetics. Kiev, "Ukraine", 2013, 335 p (en).

8. Ostapenko V. A. Energetics of molecules, atoms and nucleus. I. Physical and biophysical energetics. Kiev, "Ukraine", 2014, v.1, 370 p. (ru).

9. Ostapenko V. A. Energetics of molecules, atoms and nucleus. II. Chemical and nucleas energetics. Kiev, "Ukraine", 2015, v.2, 292 p (ru).

10. Ostapenko V. A. Research program of International Geophysical Year (IGY-1957) have ended in the Millennium beginning by discovery of the theoretical current sheets in the Nature. American Journal of Engineering Research (AJER), 2016, v.5, No.8, p.105-119.

11. Ostapenko V. A. The full calculate of discrete and continuous radiation of hydrogen plasmas for solar active formations based on the equation system of statistical equilibrium. International Organization of Scientific Research. Journal of Applied Physics (IOSR JAP), 2016, v.8, No.4, p.33-48.

12. Ostapenko V. A. The field of Lyman-alpha radiation plus current sheets and physics of various zones of the solar atmosphere. International Organization of Scientific Research. Journal of Applied Physics (IOSR JAP), 2016, v.8, No.4, p.69-79.

13. Ostapenko V. A. Eco Physical hydrogen energetics of photons and problems of ensuring the full cycle of life activity of isolated and remote settlements. Ecology and Industry, Ukraine, Kharkov, 2016, 17 p. (in press, ru).

14. Ostapenko V. A. The physical energy of the photons that used wildlife on the Earth can solve basic problems of energy and life outside the Earth. Journal of Advances in Physics (JAP), 2016, v.11, No.10, p.4093-4100 http://cirworld.com/index.php/jap/issue/view/565.

15. Ostapenko V.A. Physical energetics of photons and new technical and social possibilities of mankind. International Organization of Scientific Research. Journal of Engineering (IOSR JEN), 2016, v.6, No.8, p.1220. http://www.iosrjen.org/Papers/vol6_issue8/Version-1/B068011220.pdf.

16. Ostapenko V. A. The convective plasma turbulence changes drastically the way radiative transfer of continuous and discrete radiation of external solar layers. 2016, 13 p., (in press), "Dr. Manal" <scirea_one@ scirea.org>

17. Ostapenko V. A. Radiative and convective transfer of energy out of the star core examined with the theory of macro turbulent plasma may to change of internal zones and evolution time of stars. 2016, 13 p., (in press), "Dr. Manal"<scirea_one@scirea.org> 


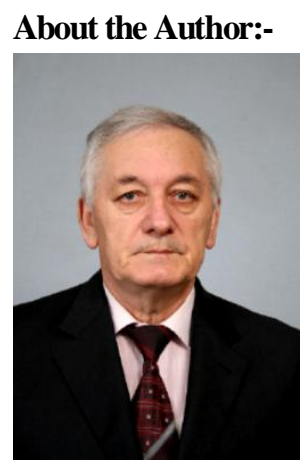

Valery A. Ostapenko (Остапенко Валерий Алексеевич) was born January 15, 1944 (December 5, 1943 in fact) in the rural family in the village Gluhovichi Bragin' district of Gomel region. Work biography started in 1960 of building construction of Svetlogorsk chemistry industry (Gomel region).

Education: Kiev State T. G. Shevchenko University (1963-1968), majoring in Astrophysics (Physics of active processes in the solar plasma). From 1968 to 1970 he served in the Soviet army troops air defense (Senior Lieutenant-engineer).

From 1970 to 2000 he worked at the scientific positions of officer to Deputy Director of the Astronomical Observatory of the Kiev State T. G. Shevchenko University. From January 1, 2001, to October 31, 2011 worked the head of sector at the Institute of innovative technologies and content of Education Ministry of education and science of Ukraine, is a representative of the Ministry in scientificmethodical commissions of Universities with training areas 0504 "Metallurgy", 0505 "Engineering Mechanics" and "0506"Energy".

Author of near 130 publications, including 5 monographs on active processes in hydrogen plasma with magnetic field, in which there are a number of discoveries (the current sheets in the Nature, and the new energetics - the physical energetics of photons). This energetics provides the potential possibilities of ecological operation with the organics as well as $\mathrm{CO}_{2}(\mathrm{CO})$ to ensure the principal aspects of the full cycle of life, without the use of oxygen and fossil fuels.
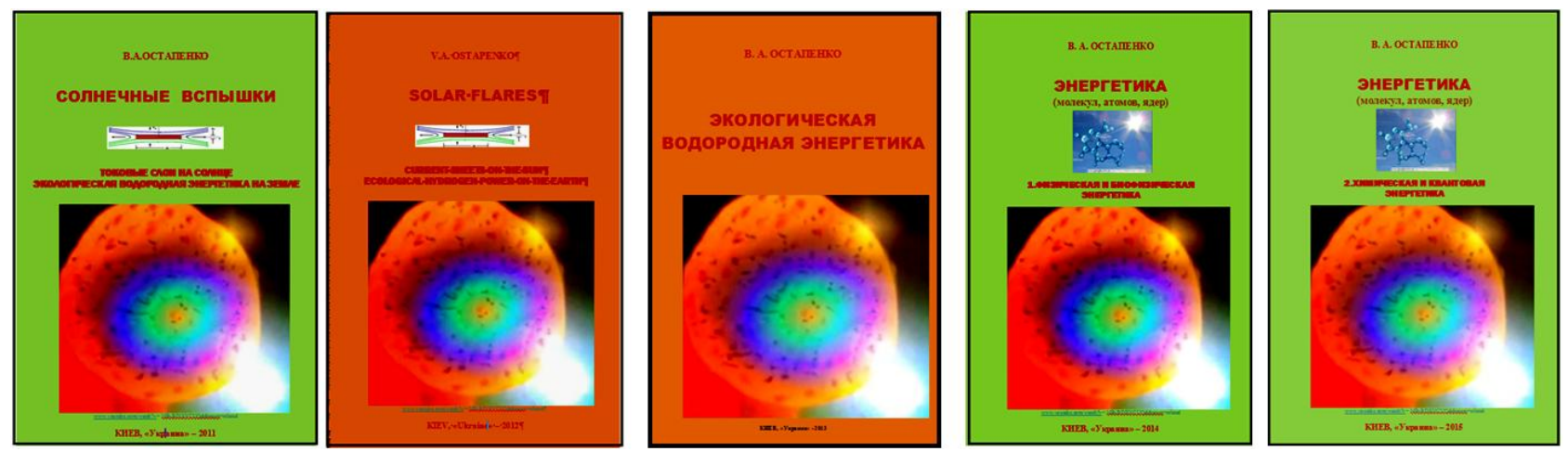

The theoretical and experimental bases of hydrogen and EFhEF energetics are written in details in the 5 monographs in Ukraine of 2011-2015 and detailed short on separate problems in the NASA periodical journals of 2016. 